\title{
Limited value of chest radiography in predicting aetiology of lower respiratory tract infection in general practice
}

\author{
A Willy Graffelman, Francois EJA Willemssen, Harmine M Zonderland, \\ Arie Knuistingh Neven, Aloys CM Kroes and Peterhans J van den Broek
}

\author{
ABSTRACT \\ Background \\ In patients with lower respiratory tract infection (LRTI), \\ changes on chest radiography are rare but poorly \\ characterised, especially in general practice. \\ Aim \\ To describe the range of findings on chest radiographs \\ and the associations between these findings and the \\ aetiology of LRTI. \\ Design of study \\ A prospective observational study. \\ Setting \\ General practices in the Leiden region, The \\ Netherlands. \\ Method \\ Adult patients with a defined LRTI were included. \\ Standard medical history and physical examination \\ were performed. Sputum, blood, and throat swabs \\ were collected for diagnostic tests. Chest X-ray \\ findings were assessed in relation to the aetiology. \\ Results \\ An abnormality on the chest X-ray was observed in 72 \\ (55\%) patients. Forty-five patients (35\%) had changes \\ due to infection, and 26 (20\%) due to pneumonia. \\ Pathogens were detected in 84 patients $(33$ single \\ bacterial, 43 single viral, and 8 dual). Twelve (29\%) \\ patients with a bacterial infection (including dual \\ infections) compared to four (9\%) patients with viral \\ infection had pneumonia on the chest $\mathrm{X}$-ray (odds ratio \\ $[\mathrm{OR}]=4.0 ; 95 \%$ confidence interval $[\mathrm{Cl}]=1.2$ to 13.8 ). \\ Using the presence of pneumonia on chest X-ray as a \\ test to predict a bacterial infection, the positive \\ predictive value and the negative predictive value were \\ $75 \%(\mathrm{Cl}=48$ to $93 \%)$ and $57 \%(\mathrm{Cl}=45$ to $69 \%)$, \\ respectively.

\section{Conclusion} \\ Pneumonia on the chest $\mathrm{X}$-ray was found more \\ frequently in patients with a bacterial infection than in \\ patients with a viral infection. However, the sensitivity \\ and the specificity are such that pneumonia on the \\ chest X-ray is not a reliable test to discriminate \\ between bacterial and non-bacterial LRTI in the general \\ practice setting. \\ Keywords \\ adult; etiology; primary health care; radiography, \\ thoracic; respiratory tract infections.
}

\section{INTRODUCTION}

Lower respiratory tract infections (LRTIs) are very common in general practice. A study in the UK showed an incidence of 44 per 1000 adult population per year. ${ }^{1}$ Investigations into patients with LRTI in general practice showed radiographically-confirmed pneumonia in $6-39 \%$ of these patients. ${ }^{1-5}$ In patients with cough and sputum production, without abnormal findings on lung auscultation, changes on chest radiography are rare. ${ }^{6}$ In general practice the majority of patients with LRTI are treated without further investigation other than physical examination. Unfortunately prediction models based on clinical information do not reliably predict the presence of an infiltrate on a chest X-ray. ${ }^{7}$ When further investigation is performed, chest radiography is the most commonly used technique.

In a prospective study on the aetiology of LRTIs in general practice carried out by this study group, chest radiographs were performed. ${ }^{8}$ This offered the

AW Graffelman, $M D, P h D$, research physician, Department of Public Health and Primary Care; A Knuistingh Neven, MD, PhD, GP, epidemiologist, Department of Public Health and Primary Care; ACM Kroes, MD, PhD, professor of medical microbiology, Department of Medical Microbiology; PJ van den Broek, $M D, P h D$, professor of infectious diseases,

Department of Infectious Diseases, Leiden University Medical Centre, Leiden, The Netherlands. FEJA Willemssen, MD, radiologist; Department of Radiology, Erasmus $M C$, Rotterdam, The Netherlands. HM Zonderland, MD, PhD, radiologist, Department of Radiology, Academic Medical Centre, University of Amsterdam, Amsterdam,

The Netherlands.

Address for correspondence

Mrs AW Graffelman, Department of Public Health and Primary Care, Leiden University Medical Center (LUMC), PO-Box 9600, 2300 RC Leiden, The Netherlands. E-mail: a.w.graffelman@lumc.nl

Submitted: 11 June 2007; Editor's response: 28 August 2007; final acceptance: 29 November 2007.

(c)British Journal of General Practice 2008; 58: 93-97.

DOI: 10.3399/bjgp08X264054 


\section{How this fits in}

In the diagnostic process of lower respiratory tract infection (LRTI), chest

radiography is the most commonly used technique for further investigation.

However, the present study shows that the chest X-ray is not a reliable test to

discriminate between bacterial and non-bacterial LRTI.

opportunity to describe the radiological abnormalities in those patients systematically. The study questions were: what is the range of findings on chest radiographs, and what are the associations between these findings and the aetiology of LRTI in patients in general practice?

\section{METHOD}

\section{Patients}

Adult patients aged 18 years and over, consulting their GP for LRTI in the Leiden region, the Netherlands, were included between 15 November 1998 and 1 June 2001 . The definition of LRTI used for the inclusion of patients is: any abnormality on pulmonary auscultation; and at least two of the following three signs and symptoms: (a) fever $>38^{\circ} \mathrm{C}$, or fever in the past 48 hours (reported by patient); (b) dyspnoea or cough (productive or nonproductive); (c) tachypnoea, malaise, or confusion. Patients who were pregnant or had diseases that could have obstructed completion of follow-up, for instance the final period of a malignant disease, were excluded.

Patients attending the surgery, as well as patients seen on home visits were included. A researcher visited the patients at home within 24 hours after the diagnosis made by the GP. The researcher took a standard history and carried out a physical examination. Sputum samples, throat swabs and blood samples were collected for microbiological analysis. Furthermore, blood was taken for erythrocyte sedimentation rate and C-reactive protein. Patients were visited again between 10 and 14 days, at which visit a second blood sample was taken.

The management of the illness remained the GP's responsibility.

\section{Chest radiographs}

Chest radiographs of patients consulting their GP for LRTI were collected. In accordance with the study protocol, the chest radiographs (postero-anterior and lateral) were performed 5-7 days after inclusion in the study. They were performed in one of three hospitals, close to where the patient lived. Local radiologists made the first assessment during routine daily practice. A radiologist who was not informed about the results of the first assessment, re-examined the radiographs systematically afterwards. In cases of a discrepancy between the two assessments, a third radiologist was asked to judge. The aim was to reach consensus. If previous X-rays were available, they were used for comparison.

\section{Radiographic findings}

The radiological criteria for the diagnosis of pneumonia are well defined..$^{9-11}$ The presence of alveolar or nonalveolar (that is, interstitial or combined alveolar and interstitial) consolidation, cavitation, pleural effusion, air bronchogram, loss of volume, and peri-bronchial wall thickening was noted. The extent of involvement, lobar or non-lobar, was noted down, and also if one or both lungs were involved. Any abnormalities not due to infection, for example, signs of a chronic obstructive pulmonary disease (COPD), cardiomegaly, and so on, were registered as well.

Based on the description of the radiographic findings, five groups of radiographic diagnoses were evaluated:

- pneumonia;

- airways disease (peri-bronchial wall thickening without a consolidation);

- non-infectious pulmonary features (abnormality on pulmonary parenchyma not due to infection);

- non-pulmonary features (cardiomegaly and others); and

- normal (none of the above-mentioned findings present).

Four types of pneumonia were distinguished:

- bronchopneumonia (patchy consolidation, loss of volume, without air bronchogram);

- segmental pneumonia (consolidation with air bronchogram, localised predominantly in one segment);

- multifocal pneumonia (consolidation with air bronchogram, in two or more lobes); and

- interstitial pneumonia (peri-bronchial thickening and ill-defined reticulonodulair shadowing).

Signs of infection on the chest X-ray were defined as: the presence of pneumonia (all four types of pneumonia as described above), or the presence of airways disease.

\section{Aetiological classification}

The aetiological diagnosis of LRTI was based on microbiological assays. Sputum samples were used for bacterial culture, and throat swabs for viral cultures. The acute phase and convalescence blood samples for serological testing were tested in pairs. Tests were performed for adenovirus, influenza virus $A$ and $B$, parainfluenza virus 1,2 , and 3 , and respiratory syncytial virus, Mycoplasma pneumoniae, Chlamydia spp, Coxiella burnetii, and Legionella pneumophila. Polymerase chain reaction (PCR) amplification of $M$. 
pneumoniae was carried out on throat swabs; details are given in an earlier study. ${ }^{8}$ The aetiologies were classified as bacterial, viral, or dual infections (if both a bacterium and a virus were found) according to the microbiological findings. If no pathogen was found the aetiology was classified as being unknown.

\section{Statistical analysis}

Data were analysed using SPSS version 11.0 for Windows. Mean values were calculated for the numerical variables. The $\chi^{2}$ test was used to compare percentages between groups. Significance level was set at 0.05 .

For the presence of pneumonia as well as for the presence of 'signs of infection' (sum of pneumonia and airways disease) as predictors of bacterial infection, odds ratio $(\mathrm{OR})$, sensitivities, specificities, positive predictive values, and negative predictive values were calculated with $95 \%$ confidence intervals $(\mathrm{Cl})$.

\section{RESULTS}

\section{Patients}

A total of 145 patients with LRTI were included in the study. In 137 of these patients a chest radiograph was performed. Two patients considered themselves too ill, four patients refused, one had recovered, and one had forgotten to visit the hospital for chest radiography. From these chest radiographs 129 were reviewed in detail; eight went missing. The mean age of the patients was 50 years (standard deviation 14 years), 86 patients $(53 \%)$ were female, and $76(59 \%)$ were ex-or current smokers. Ex-smokers had higher mean packyears of smoking (24 years) than current smokers (20 years). Half the patients $(n=63)$ had comorbidity (from general practice medical records), predominantly cardiovascular (23\%) and/or pulmonary (19\%) diseases; six patients had both. The mean length of time between the onset of symptoms and inclusion in the study was 9 days. The mean time between inclusion and the chest X-ray was 5 days.

\section{Radiographic findings}

In the routine assessment 29 patients were diagnosed with pneumonia, of these, four were regarded as doubtful, seven as possible and 18 as confirmed pneumonia (descriptions by radiologists in local hospitals). In the re-examination, 23 patients were considered to have pneumonia. A third opinion to reach consensus was necessary in 20 out of 129 patients. Finally 26 patients (20\%) were diagnosed with pneumonia based on the chest X-ray. An abnormal chest radiograph was observed in 72 patients $(55 \%)$. The chest radiographic features of patients with LRTI are shown in Table 1. Pulmonary findings consistent with an infection were seen in 45 (35\%) patients (including airways disease and pneumonia). The most common type of pneumonia was segmental pneumonia seen in 11 patients (42\% of those with pneumonia). Pneumonia was predominantly seen in the left lower lobe (15 patients, or $58 \%$ of those with pneumonia). Three patients had pneumonia in both lungs. In patients with non-infectious pulmonary findings, signs of COPD were predominant, and in patients with non-pulmonary findings signs of cardiomegaly were the most common feature. In 18 patients more than one chest radiographic abnormality was found.

There was no significant relationship found between sex, old age, smoking, and radiographic pneumonia. Comorbidity, particularly pulmonary disease, was found on the chest X-ray in one patient with pneumonia and in 40 patients without pneumonia.

\section{Aetiological findings}

Pathogens were identified in 84 patients (65\%); 33 had a single bacterial infection, 43 a single viral infection, and eight had a dual infection. In 41 (49\%) of the patients in whom a pathogen was identified this was a bacterial micro-organism (single bacterial and dual infection). The most common bacterial pathogens found were $M$. pneumoniae and Haemophilus influenzae, and the most common virus was influenza virus type $A$. In the 129 patients studied, the distribution of bacterial pathogens was $M$. pneumoniae $10 \%, H$. influenzae $9 \%$, Streptococcus pneumoniae $7 \%$, and other bacterial pathogens $5 \%$, and the distribution of viral pathogens was influenza virus (A and B) $28 \%$, and others $9 \%$.

\section{Relationship between aetiology and chest radiographic findings}

Pneumonia on the chest X-ray was found in $12(29 \%)$ out of 41 patients with a bacterial infection (including the dual infections), in 4 (9\%) out of 43 patients with a

\section{Table 1. Distribution of radiographic and aetiological findings in 129 patients with lower respiratory tract infection.}

\begin{tabular}{lccccc} 
& \multicolumn{5}{c}{ Aetiology } \\
\cline { 2 - 6 } & Bacterial & Viral & Dual & Unknown & Total \\
Radiographic findings & $n=33$ & $n=43$ & $n=8$ & $n=45$ & $n=129$ \\
\hline Pneumonia & 10 & 4 & 2 & 10 & 26 \\
Segmental pneumonia & 3 & 1 & 1 & 6 & 11 \\
Bronchopneumonia & 3 & 2 & 1 & 3 & 9 \\
Interstitial pneumonia & 1 & - & - & 1 & 2 \\
Multi focal pneumonia & 3 & 1 & - & - & 4 \\
\hline Airways disease & 5 & 5 & 2 & 7 & 19 \\
\hline Non-infectious & 7 & 8 & 1 & 10 & 26 \\
pulmonary features & & & & & 19 \\
\hline Non-pulmonary features & 5 & 5 & 2 & 7 & 57 \\
\hline Normal chest X-ray & 11 & 25 & 2 & 19 &
\end{tabular}

Values are number of cases. Eighteen patients had more than one radiographic finding. 


\begin{tabular}{|c|c|c|}
\hline & \multicolumn{2}{|c|}{ Radiographic findings } \\
\hline & $\begin{array}{l}\text { Pneumonia, } \\
\%(95 \% \mathrm{Cl})\end{array}$ & $\begin{array}{c}\text { Signs of infection, } \\
\%(95 \% \mathrm{Cl})\end{array}$ \\
\hline \multicolumn{3}{|c|}{ At observed prevalence $(41 / 84)$} \\
\hline Sensitivity & 29 (16 to 46$)$ & 46 (31 to 63$)$ \\
\hline Specificity & 91 (75 to 97$)$ & 79 (64 to 90$)$ \\
\hline Positive predictive value & 75 (48 to 93$)$ & $68(48$ to 84$)$ \\
\hline Negative predictive value & 57 (45 to 69 ) & $61(47$ to 74$)$ \\
\hline \multicolumn{3}{|c|}{ At maximum prevalence $(86 / 129)^{a}$} \\
\hline Positive predictive value & 85 (65 to 96$)$ & 80 (65 to 90$)$ \\
\hline Negative predictive value & 38 (29 to 47$)$ & 41 (30 to 52$)$ \\
\hline \multicolumn{3}{|c|}{ At minimal prevalence $(41 / 129)^{a}$} \\
\hline Positive predictive value & 46 (27 to 65$)$ & $42(28$ to 58$)$ \\
\hline Negative predictive value & 72 (63 to 81$)$ & 74 (63 to 83$)$ \\
\hline
\end{tabular}

aFor the 'maximum prevalence' all aetiological unknown cases were considered as of bacterial origin, and for the 'minimal prevalence' these cases were considered as of viral origin.

viral infection, and in $10(22 \%)$ out of 45 patients with an unknown aetiology. The distribution of radiographic findings and aetiology is shown in Table 1. The difference between the proportions of pneumonia on the chest X-ray in patients with bacterial infections ( $n$ $=41)$ compared to patients with viral infections $(n=43)$ was significant $(P=0.02)$, with $\mathrm{OR}=4.0(95 \% \mathrm{Cl}=1.2$ to 13.8). Findings on the chest $\mathrm{X}$-ray related to infection (pneumonia and airways disease) were found in $19(46 \%)$ of the patients in whom bacteria were detected, in nine (21\%) patients with a virus detected, and in 17 (38\%) patients in whom no pathogen was detected $(30 \%$ in the combined group of viral and unknown infection). The difference between the proportions of signs of infection on the chest X-ray in patients with bacterial infections $(n=41)$ compared to patients with viral infections $(n=43)$ was significant $(P$ $=0.01)$, with $\mathrm{OR}=3.3(95 \% \mathrm{Cl}=1.3$ to 8.5$)$. The proportions of non-infectious pulmonary findings and non-pulmonary findings were similar in patients with bacterial infections, viral infections, or infections of unknown aetiology.

The chest X-ray was examined as a test for the prediction of bacterial infections. This examination was performed with the 84 patients in whom pathogens were detected. The test characteristics are shown in Table 2.

Because the aetiology was unknown in $35 \%$ of the patients, the predictive values for the outside limits of the prevalence of bacterial infection were calculated. When one assumes that all patients in whom no pathogen was found have a viral infection (minimal bacterial prevalence of $32 \%$ ), the positive predictive value for a patient with pneumonia on the chest $\mathrm{X}$-ray to have bacterial infection was $46 \%$. Assuming all unknown cases were of bacterial origin (maximal bacterial prevalence $67 \%$ ), the positive predictive value to have bacterial infection when an infiltrate on the chest X-ray is present was $85 \%$. When the same is done for the broader category of 'signs of infection' on the chest $\mathrm{X}$-ray, the positive predictive values at minimal and maximal prevalence of bacterial infection are $42 \%$ and $80 \%$, respectively.

\section{DISCUSSION}

\section{Summary of main findings}

Patients who consulted their GP with symptoms of a LRTI and who had abnormalities on auscultation of the chest, showed a variety of chest radiographic abnormalities. Most common were the changes that were attributed to pneumonia and to non-infectious pulmonary features. Patients with a radiographically confirmed pneumonia had a fourfold higher chance of having a bacterial infection than a viral infection, compared to patients without pneumonia on the chest $\mathrm{X}$-ray. If the chest X-ray was used as a test for the prediction of bacterial infections, the chance of a bacterial infection changed from about even chances to three-quarters.

\section{Comparison with existing literature}

The present observations of $46 \%$ 'signs of infection', on the chest X-rays of patients with bacterial infection, and $30 \%$ in patients with viral or unknown infection is somewhat higher than the observation by Macfarlane et al who found chest radiographic changes consistent with infection in $25 \%$ of the patients with bacterial/atypical pathogens and in $16 \%$ of the patients with viral infection or no pathogens identified. ${ }^{1}$ This may be attributed to the difference in inclusion criteria. In contrast to the present study, in the study by Macfarlane et al, 'abnormalities on auscultation' was not a prerequisite, and comorbidity was excluded. ${ }^{1}$ So, the somewhat higher percentages observed in the present study could match with a less healthy population.

The present findings of $29 \%$ pneumonia on the X-ray in patients with bacterial infection and $9 \%$ in patients with viral infection are comparable with the findings of Hopstaken et al, $22 \%$ and $18 \%$ pneumonia, respectively. ${ }^{12}$

\section{Strengths and the limitations of the study}

The present study describes the radiological abnormalities and systematically investigates the association between these findings and the aetiology of LRTI in patients in general practice.

Unfortunately, $54 \%$ of the patients with a bacterial or dual infection had no signs of infection (pneumonia or airways disease) on the chest X-ray, for which several reasons can be postulated. The infection may have been minor and may not have affected the lung tissue. It is also possible that the sensitivity of the chest X-ray was too low to detect the infection. 
Chest radiography was used as the standard reference to identify pneumonia, because of its low cost and general accessibility. Both the low sensitivity and the inter-observer variability play a role in its reliability. Because of the low sensitivity, some of the infiltrates could have been missed. To reduce the possibility of false-positive or false-negative test results, the chest Xrays were re-examined. The chest $\mathrm{X}$-rays were taken about 5 to 7 days after inclusion in the study, the mean time was 5 days. Another study by Macfarlane et al showed that abnormalities generally persist for a fairly long time; 1 week after the diagnosis of pneumonia only $5-10 \%$ of the abnormalities had resolved..$^{13}$

However, a study by Mittl, et al showed complete resolution in $50 \%$ of patients after 2 weeks. ${ }^{14}$ This resolution of pneumonia was related to age and was less rapid in older patients. The patient population in the current study, mean age of 50 years, is somewhat older than that in Mittl's population, with its mean age of 40 years. The relevance of the present findings that patients already had symptoms for 9 days on average before inclusion, which was in fact 14 days before the chest X-ray was performed, remains unclear. The studies by Macfarlane et al and Mittl et al did not show data on this subject. ${ }^{13,14}$ Nevertheless, in the present study, the diagnosis of pneumonia may have been missed in some patients.

\section{Implications for clinical practice}

If pneumonia on the chest X-ray had been used as a diagnostic test for the presence of bacterial infection, that is, as indication for the start of antibiotic treatment, in the observed population with known aetiology ( $n=$ 84), the following would have happened: 16 patients would have been treated with antibiotics; of these, 12 indeed had bacterial infection and would have been treated correctly. Of the 68 patients who would not have received an antibiotic, 29 had bacterial infection and would have been treated incorrectly, considering bacterial LRTI an indication for antibiotic therapy. If 'signs of infection' on the chest X-ray had been the criterion, the following would have happened: 28 patients would have been treated with antibiotics; of these, 19 indeed had bacterial infection and would have been treated correctly. Of the 56 patients who would not have received an antibiotic, 22 had bacterial infection and would have been treated incorrectly.

Although the reduction of antibiotic use that can be achieved by using the chest $\mathrm{X}$-ray as a criterion for antibiotic treatment is high $(80 \%$ and $66 \%$ using pneumonia or signs of infection as indication, respectively) compared with the antibiotic treatment of 128 of the 129 patients with LRTI in the present study population, the proportions of untreated patients with bacterial infection are unacceptable in this population of severely ill patients.
In conclusion, the present study shows that pneumonia or signs of infection on the chest $X$-ray were found more frequently in patients with bacterial infections than in those with viral infections. The sensitivity and the specificity are such that pneumonia on the chest X-ray is not a reliable test to discriminate between bacterial and non-bacterial LRTI in the general practice setting.

\section{Funding body}

This work was made possible by a grant from the European Commission Framework V (grant number QLK2-CT-200000294) and from Pfizer

\section{Ethical approval}

The Medical Ethics Committee of the Leiden University Medical Centre (reference protocol number P140/98)

\section{Competing interests}

The authors have stated that there are none

\section{Acknowledgements}

We wish to thank the patients and GPs who participated in the study. We would also like to thank Mrs $\mathrm{H}$ Bolk-Lucieer for correction of English and Professor WJJ Assendelft (Department of Public Health and Primary Care, LUMC Leiden) for his constructive criticism

\section{Discuss this article}

Contribute and read comments about this article on the Discussion Forum: http://www.rcgp.org.uk/bjgp-discuss.

\section{REFERENCES}

1. Macfarlane J, Holmes W, Gard P, et al. Prospective study of the incidence, aetiology and outcome of adult lower respiratory tract illness in the community. Thorax 2001; 56(2): 109-114.

2. Macfarlane JT, Colville A, Guion A, et al. Prospective study of aetiology and outcome of adult lower-respiratory-tract infections in the community. Lancet 1993; 341(8844): 511-514.

3. Lieberman D, Lieberman D, Korsonsky I, et al. A comparative study of the etiology of adult upper and lower respiratory tract infections in the community. Diagn Microbiol Infect Dis 2002; 42(1): 21-28.

4. Woodhead MA, Macfarlane JT, Mc Cracken JS, et al. Prospective study of aetiology and outcome of pneumonia in the community. Lancet 1987; 1(8534): 671-674.

5. Hopstaken RM, Muris JWM, Knottnerus JA, et al. Contributions of symptoms, signs, erythrocyte sedimentation rate, and C-reactive protein to a diagnosis of pneumonia in acute lower respiratory tract infection. $\mathrm{BrJ}$ Gen Pract 2003; 53(490): 358-364.

6. Puhakka T, Lavonius M, Varpula M, et al. Pulmonary imaging and function in the common cold. Scand J Infect Dis 2001; 33(3): 211-214.

7. Graffelman AW, le Cessie S, Knuistingh Neven A, et al. Can history and exam alone reliably predict pneumonia? J Fam Pract 2007; 56(6): 465-470.

8. Graffelman AW, Knuistingh Neven A, le Cessie S, et al. Pathogens involved in lower respiratory tract infections in general practice. Br J Gen Pract 2004; 54(498): 15-19.

9. Katz DS, Leung AN. Radiology of pneumonia. Clin Chest Med 1999; 20(3): 549-562.

10. Goodman PC, Wilson AG, Armstrong P, Murray JF. Pulmonary infection in adults. In: Grainger RG, Allison D, Carty H (eds). Diagnostic radiology. A textbook of medical imaging. London: Churchill Livingstone, 2001: 377-417.

11. Lynch D. Large airway disease. In: Grainger RG, Allison D, Carty H (eds). Diagnostic radiology. A textbook of medical imaging. London: Churchill Livingstone, 2001: 419-438.

12. Hopstaken RM, Stobberingh EE, Knotnerus JA, et al. Clinical items not helpful in differentiating viral from bacterial lower respiratory tract infections in general practice. J Clin Epidemiol 2005; 58(2): 175-183.

13. Macfarlane JT, Miller AC, Roderick Smith WH, et al. Comparative radiographic features of community acquired legionnaires disease, pneumococcal pneumonia, mycoplasma pneumonia, and psittacosis. Thorax 1984; 39(1): 28-33.

14. Mittl RL, Schwab RJ, Duchin JS, et al. Radiographic resolution of community-acquired pneumonia. Am J Respir Crit Care Med 1994; 149(3 Pt 1): 630-635. 